\title{
Plasma electrolytes in elderly patients taking fixed combination diuretics
}

\author{
A.J. Bayer, R. Farag, S. Browne and M.S.J. Pathy \\ Department of Geriatric Medicine, University Hospital of Wales, Heath Park, Cardiff CF4 4XW, UK.
}

\begin{abstract}
Summary: Plasma potassium and sodium concentrations were measured in a group of elderly patients taking maintenance thiazide diuretic therapy alone, with a potassium supplement or in combination with a potassium sparing diuretic. Fixed dose combinations of a thiazide and potassium sparing diuretic did not significantly reduce the prevalence of hypokalaemia and the combination of amiloride-hydrochlorothiazide was associated with a disproportionate number of cases of hyponatraemia. The desirability of the current widespread use of fixed dose combination diuretics over less expensive single agents is questioned.
\end{abstract}

\section{Introduction}

Fixed dose combination diuretics, containing both a thiazide and a potassium sparing diuretic, are widely used to aid compliance and reduce the risk of hypokalaemia. Such combined preparations, however, have many disadvantages. They are inflexible and expensive and cannot be relied upon to control plasma electrolytes (Drugs and Therapeutics Bulletin, 1985): the elderly appear particularly susceptible to such disturbances and this has led to a recent suggestion that a combination diuretic should not be in general use in older patients (Millson et al., 1984).

The present study reports the prevalence of electrolyte disturbance among elderly patients taking long-term, thiazide diuretic therapy alone, or in combination with a potassium sparing agent.

\section{Patients and methods}

Plasma potassium and sodium concentrations were measured in 197 consecutive patients (mean age 78.3 years, range 63-101; 79 males, 118 females) newly admitted to one of three acute geriatric units and who had been taking thiazide diuretics as long-term maintenance therapy for heart failure or hypertension. In all patients, the type and dose of diuretic and potassium supplement when prescribed had not been recently changed. Of the $68(35 \%)$ patients taking a thiazide diuretic alone, 41 had also been prescribed a potassium supplement. In 18 patients this was combined with the diuretic and in 23 was taken separately. Of the

Correspondence: A.J. Bayer, M.B., B.Ch.

Accepted: 15 August 1985
$129(65 \%)$ patients taking a thiazide combined with a potassium sparing diuretic, 93 were on a triamterenehydrochlorothiazide combination ('Dyazide') and 36 were on an amiloride-hydrochlorothiazide combination ('Moduretic').

\section{Results}

Figure 1 compares the plasma potassium concentrations of patients taking a thiazide diuretic alone, with a potassium supplement, or in combination with amiloride or triamterene. Mean plasma potassium concentrations in each diuretic group were similar (overall means, in both sexes, $3.9 \pm 0.7 \mathrm{mmol} / \mathrm{l}$ ). Hypokalaemia (plasma potassium less than $3.0 \mathrm{mmol} / \mathrm{l}$ ) was present in $13(7 \%)$ patients, 7 of whom were taking a combination product and 4 were supposedly taking a potassium supplement. Significant hyperkalaemia $(6.4 \mathrm{mmol} / \mathrm{l})$ was present in only one patient who was taking an amiloride-hydrochlorothiazide combination. He was known to have chronic renal impairment and died suddenly within 24 hours of admission to hospital.

The plasma sodium concentrations of each patient are shown in Figure 2. Mean plasma sodium concentration in those taking amiloride-hydrochlorothiazide $(133.3 \pm 5.1 \mathrm{mmol} / \mathrm{l})$ was significantly lower (Student's $t$ test for unpaired data, $P<0.002$ ) than those taking triamterene-hydrochlorothiazide $(136.3 \pm 4.2 \mathrm{mmol} /$ 1) or a thiazide with or without potassium supplement $(136.7 \pm 4.8 \mathrm{mmol} / \mathrm{l})$. Similarly, hyponatraemia (plasma sodium less than $130 \mathrm{mmol} / \mathrm{l}$ ) was present in $11(31 \%)$ of patients taking amiloride-hydrochlorothiazide, but only $4(4 \%)$ of those taking triamterene-

(C) The Fellowship of Postgraduate Medicine, 1986 


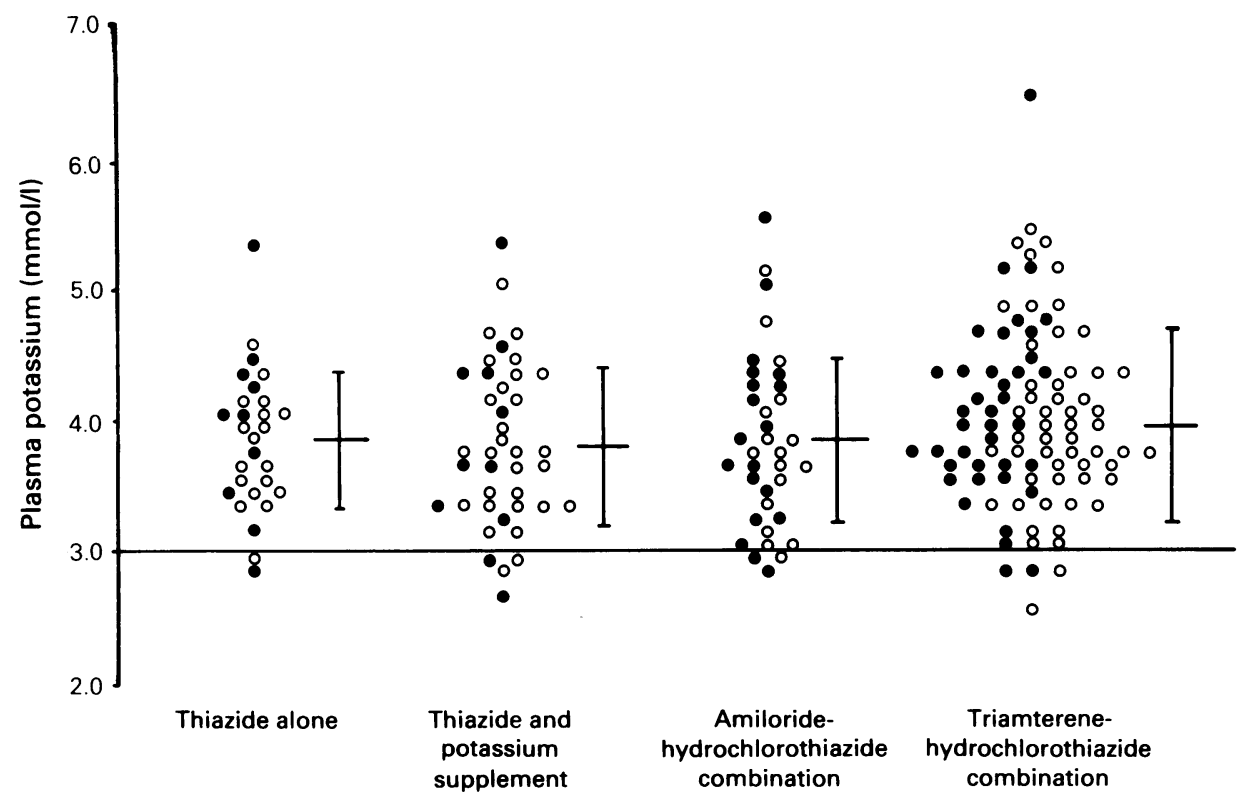

Figure 1 Plasma potassium in patients taking thiazide diuretics (with mean \pm s.d.) (O) male; $(O)$ female.

hydrochlorothiazide and $6(9 \%)$ of those taking a thiazide diuretic alone.

There was no significant relationship between the degree of hyponatraemia and plasma urea. Fourteen $(67 \%)$ of the hyponatraemic patients had symptoms (weakness, drowsiness, confusion, unsteadiness and nausea) which improved once plasma sodium had returned to the normal range, but in no case was the low serum sodium the cause for the patient's admis sion to hospital.

Age and sex had no significant influence on the? prevalence or severity of electrolyte disturbances, nor

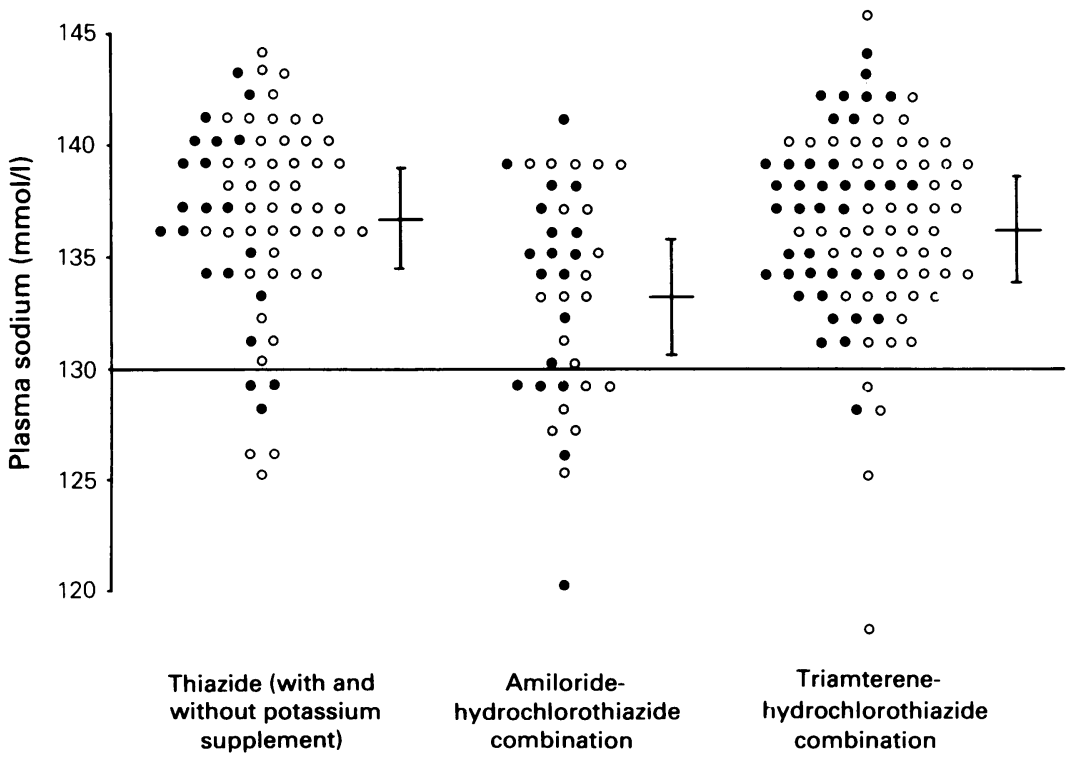

Figure 2 Plasma sodium in patients taking thiazide diuretics (with mean \pm s.d.) (O) male; (O) female. 
was there any difference in electrolyte values between those with hypertension or cardiac failure.

\section{Discussion}

Diuretics are among the drugs most commonly prescribed to old people at home (Law \& Chalmers, 1976) and those requiring hospital admission (Williamson \& Chopin, 1980). Many factors should influence the choice of diuretic for maintenance therapy (Orme \& Green, 1979), but the present series confirms the current widespread use of fixed dose combination products in the elderly. The argument for their routine use centres on the potential risk of hypokalaemia in this age group, yet our findings would suggest, as did those of Krakauer \& Lauritzen (1978), that the elderly are not especially prone to thiazide-induced hypokalaemia. Despite their theoretical advantages, combination diuretics did not reduce the prevalence of hypokalaemia when compared to patients taking thiazides alone or to those prescribed a thiazide and potassium supplement.

Although no recent changes in dosage of diuretic or potassium supplement had been made, our finding of similar plasma potassium concentrations in each group may reflect previous adjustments following measurement of plasma electrolytes. As most patients, however, had previously been solely treated by their general practitioners this would seem unlikely. Furthermore, the apparent failure of potassium supplements to influence plasma potassium concentrations in patients on long-term thiazide diuretics is well recognized: to have a useful effect they must be given in a dose of at least $24 \mathrm{mmol} /$ day (Beeley, 1980) and few patients in the present study had therefore been prescribed adequate doses. Potassium sparing diuretics, although more effective in preventing hypokalaemia, are not always successful (Antcliffe et al., 1972; Penhall \& Frewin, 1980) and the present series also confirms the risk of producing dangerous hyperkalaemia in elderly patients with impaired renal function (Jaffey \& Martin, 1981).

\section{References}

ANTCLIFF, A.C., BEEVERS, D.G., HAMILTON, M. \& HARPUR, J.E. (1972). Amiloride hydrochloride combined with hydrochlorothiazide in the control of hypertension and plasma potassium levels. British Journal of Clinical Practice, 26, 413.

BEELEY, L. (1980). When do patients on diuretics need potassium supplement? Adverse Drug Reaction Bulletin, 84, 304.

BEERMANN, B. \& GROSCHINSKY-GRIND, M. (1977). Pharmacokinetics of hydrochlorothiazide in man. European Journal of Clinical Pharmacology, 12, 297.
Hyponatraemia is a less well recognized side effect of maintenance thiazide diuretic therapy, but there have been recent reports of its frequency in elderly patients and its association with considerable morbidity and mortality (Sunderam \& Mankikar, 1983; Rozkovec \& Marshall, 1983). The combination of amiloride-hydrochlorothiazide has been specifically implicated (Brooks \& Ritch, 1983; Millson et al., 1984) and in the present series nearly one third of users of this preparation were significantly hyponatraemic. We could not confirm a recent suggestion that elderly hypotensive women are particularly prone to diureticinduced hyponatraemia (Cogan \& Abramow, 1983).

Although the dose response effect of thiazides on plasma electrolytes appears relatively flat (Beerman \& Groschinsky-Grind, 1977), it is possible that the differences between the groups studied may have been due to different thiazide dosage. In terms of hydrochlorothiazide equivalent, mean dosages in the thiazide alone and amiloride and triamterene combination groups were, 41,48 and $33 \mathrm{mg}$. Most patients were taking one diuretic tablet daily, but the dose of hydrochlorothiazide in the amiloride combination studied is twice that in the triamterene combination and greater than the equivalent diuretic dose in most other single thiazide tablets. Electrolyte disturbance may therefore be less if prescribers give greater regard to the actual dose of diuretic prescribed, rather than apparently regarding single diuretic tablets as having equal potency. The availability of a lower dose amiloride-hydrochlorothiazide combination ('Moduret-25') may also be of help.

We conclude that in the present study, the use of fixed dose combination thiazide and potassium sparing diuretics did not reduce the prevalence of hypokalaemia and the combination of amiloride-hydrochlorothiazide, in the formulation studied, was associated with a disproportionate number of cases of hyponatraemia. The advantages of the current widespread use of combination diuretics over much less expensive single agents must therefore be seriously questioned.

BROOKS, R.W.S. \& RITCH, A.E.S. (1983). New drugs: modern diuretic treatment. British Medical Journal, 286, 1971.

COGAN, E. \& ABRAMOW, M. (1983). Diuretic induced hyponatraemia in elderly hypertensive women. Lancet, ii, 1249.

Drugs and Therapeutics Bulletin. (1985). Potassium-sparing diuretics - when are they really needed? 23, 17.

JAFFEY, L. \& MARTIN, A. (1981). Malignant hyperkalaemia after amiloride/hydrochlorothiazide treatment. Lancet, i, 1272.

KRAKAUER, R. \& LAURITZEN, M. (1978). Diuretic therapy 
and hypokalaemia in geriatric out-patients. Danish Medical Bulletin, 25, 126.

LAW, R. \& CHALMERS, C. (1976). Medicines and elderly people: a general practice survey. British Medical Journal, 1, 565.

MILLSON, D., BORLAND, C., MURPHY, P. \& DAVISON, W. (1984). Hyponatraemia and Moduretic. British Medical Journal, 289, 1308.

ORME, M.L.E. \& GREEN, G.J. (1979). Diuretics: which for what? British Journal of Hospital Medicine, 21, 272.

PENHALL, R.K. \& FREWIN, D.B. (1980). Plasma potassium levels in hypertensive patients receiving fixed-combination diuretic therapy. Medical Journal of Australia, 1, 376.

ROZKOVEC, A. \& MARSHALL, A.J. (1983). New drugs: modern diuretic treatment. British Medical Journal, 286, 1971.

SUNDERAM, S.G. \& MANKIKAR, G.D. (1983). Hyponatraemia in the elderly. Age and Ageing, 12, 77.

WILLIAMSON, J. \& CHOPIN, J.M. (1980). Adverse reactions to prescribed drugs in the elderly: A multicentre investigation. Age and Ageing, 9, 73. 